\title{
Erratum
}

\section{Chaotic Motion of a Rigid Rotor ${ }^{1}$}

\author{
F. M. El Sabaa ${ }^{2}$ \\ Received August 2, 1996
}

On p. 1870, the first line of equation (2) should read

$$
p=\dot{\psi} \sin \theta \sin \phi+\dot{\theta} \cos \phi
$$

On p. 1870, the third line of equation (2) should read

$$
r=\dot{\psi} \cos \theta+\dot{\phi}
$$

On p. 1871, the last line of equation (3) should read

$$
\left.\left.+B(\dot{\phi} \sin \theta \cos \phi+\dot{\theta} \sin \phi)^{2}+C(\dot{\psi} \cos \theta+\dot{\phi})^{2}\right]-V(\theta, \phi)\right]
$$

On p. 1872, equation (7) should read

$$
D^{-1}=\left(A \sin ^{2} \phi+B \cos ^{2} \phi\right) \sin ^{2} \theta+C \cos ^{2} \theta
$$

'This paper originally appeared in International Journal of Theoretical Physics, 9, 18691883 (1994).

${ }^{2}$ Department of Mathematics, Faculty of Education, Roxy, Cairo, Egypt. 